\title{
Modal reflectivity in finite-depth two-dimensional photonic-crystal microcavities
}

\author{
Brian D’Urso, Oskar Painter, John O'Brien, Tom Tombrello, Amnon Yariv, and Axel Scherer \\ California Institute of Technology, Electrical Engineering/Applied Physics, MS 200-36, Pasadena, California 91125
}

Received June 4, 1997; revised manuscript received October 22, 1997

\begin{abstract}
We present finite-difference time-domain calculations of the $Q$ factor for an optical microcavity defined by a slab waveguide and two-dimensional photonic-crystal end mirrors. The effect of the finite depth of the photonic crystal on the cavity's optical modes is examined. From these calculations, we can optimize the performance of the photonic-crystal mirrors and determine the loss mechanisms within optical cavities defined by these structures. The $Q$ of the cavity modes is shown to be strongly dependent on the depth of the holes defining the photonic crystal, as well as the refractive index of the material surrounding the waveguide core. (C) 1998 Optical Society of America [S0740-3224(98)01803-7]

OCIS codes: $230.4000,260.2110$.
\end{abstract}

\section{INTRODUCTION}

Two-dimensional (2D) photonic band-gap crystals have been proposed and demonstrated in both active and passive semiconductor devices. ${ }^{1-7}$ Applications of these structures range from simple passive elements, such as filters and polarizers, to ultrasmall cavity lasers and single-mode LED's, with the tantalizing possibilities of inhibited spontaneous emission and improved efficiencies of light emitters. ${ }^{1}$ Realistically, the finite dimensions of $2 \mathrm{D}$ photonic crystals have to be addressed in designing and optimizing devices incorporating photonic crystals. Technologies such as silicon-on-insulator and the emergence of steam oxidation of AlAs has recently made possible the definition of the high refractive-index contrast needed to confine light within a finite plane.

In light of these new technological developments, we present in this paper numerical calculations of the threedimensional (3D) field distribution of guided modes in a waveguide resonator defined by $2 \mathrm{D}$ photonic-crystal end mirrors. We have determined the effect of the finite depth of $2 \mathrm{D}$ photonic crystals in three specific cases: (a) in a standard semiconductor slab-waveguide structure, (b) in a waveguide whose bottom cladding has been removed and replaced by air, and (c) in a waveguide whose semiconductor cladding has been completely removed and replaced by air on the top and bottom of the core. In all three cases the end mirrors are a $2 \mathrm{D}$ hexagonal lattice of circular air holes, in which the depth of the holes is varied to show the influence of the etch depth on the cavity $Q$ 's.

\section{DESCRIPTION OF PROBLEM}

A variety of methods for fabricating photonic band-gap materials at optical wavelengths in semiconductors have been proposed. ${ }^{8-10}$ In this paper we focus on photonic crystals fabricated by etching air holes into the semiconductor. The results, however, can be applied to other types of microfabricated photonic crystal. At present, etch anisotropy and nonradiative surface recombination technologically limit the implementation of photonic crystals in active semiconductor light emitters. To fabricate $2 \mathrm{D}$ crystals, we use electron-beam lithography, followed by reactive ion etching to transfer the $2 \mathrm{D}$ mask pattern into the semiconductor waveguide.

The incorporation of these photonic crystals into active optical devices presents a variety of challenges. The inplane dimensions for optical photonic band-gaps are of the order of hundreds of nanometers. Conversely, the depth of most low-loss lasers extends at least 1-2 $\mu \mathrm{m}$ deep, and so high aspect ratios in the etched hole structures are required, as shown in the scanning-electron micrographs of Fig. 1.

Another difficulty in fabricating active devices that utilize photonic-crystal microcavities is the creation of large surface-to-volume ratios in the active region. The associated nonradiative surface recombination, which is particularly severe in the GaAs-AlGaAs material system, ${ }^{11,12}$ limits the application of photonic crystals in active GaAs semiconductor devices. To address both of these technological limitations, it is desirable to perforate the waveguide only partially with holes in order to fabricate high- $Q$, low-loss microcavities. Clearly, the effects of varying hole depth in the fabrication of these shallow structures cannot be ignored in determining device characteristics.

In designing photonic band-gap crystal microcavities for use in semiconductor lasers (and resonant enhancement LEDs) one must also be careful not to presume a low-loss cavity simply from band-gap calculations of an infinite lattice. In lasers the important parameter is modal reflectivity, not the total reflected power from a mirror. For 2D and 3D photonic-crystal mirrors, the directions in which the power is reflected are equally important. Since these multidimensional photonic-crystal lattices are composed of Bragg planes at many different angles, they are able to reflect incident electromagnetic waves in a variety of directions and in a complex manner. An incident mode may have all its power reflected, but the reflected power can be distributed among many other 

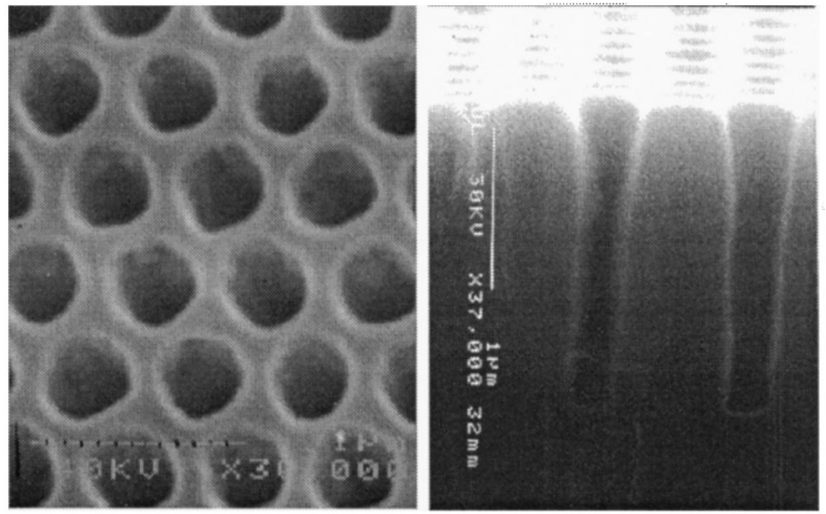

Fig. 1. 2D hexagonal array of holes etched $2 \mu \mathrm{m}$ deep into AlGaAs laser structure. The holes are $300 \mathrm{~nm}$ in diameter, and the spacing is $500 \mathrm{~nm}$.

modes. Thus it is the modal reflectivity that determines the $Q$ of a given cavity mode. ${ }^{13}$ Another important parameter, besides the $Q$, is the mode volume. To modify spontaneous emission it will be necessary to fabricate an optical cavity that supports one or, at most, a few optical, high- $Q$, modes. This requires an optical volume with dimensions of the order of $\lambda /(2 n) .{ }^{14-16}$ Extended modes, such as the modes in a distributed-feedback laser cavity, do not suffice, as the modal reflectivity is high, but the optical volume is large.

\section{DESCRIPTION OF THE MODEL CALCULATIONS}

In our analysis we consider an in-plane resonator formed between two 2D hexagonal-lattice photonic crystals. Figure 2 shows a top view along with a cross section of the structure. A three-layer slab waveguide is used to provide vertical confinement of the optical mode. The $Q$ of the resonator mode is calculated, from which a modal reflectivity is inferred. This is done for various penetration depths of the photonic crystal into the waveguide structure. The core of the waveguide is modeled with a refractive index $n_{\text {core }}=3.3$, whereas the cladding index is defined as either $n_{\text {clad }}=3.0$ or $n_{\text {clad }}=1.0$, depending on the geometry analyzed. 2D photonic band-gap calculations of periodic crystals with infinite depth and extent were performed by Joannopoulos et al. at MIT, where a bandgap map was generated that outlines the gap frequency versus crystal geometry. ${ }^{17}$ The photonic crystal mirrors in our analysis comprise a hexagonal pattern of cylindrical holes extending into the waveguide. The ratio of hole radius to hole spacing, $r / a$, and the hole spacing to wavelength ratio, $a / \lambda_{0}$, were chosen as 0.4 and 0.28 , respectively. These ratios provide a $2 \mathrm{D}$ band-gap for the infinite crystal and correspond to the design used in our edge-emitting laser fabrication. ${ }^{3}$ In our calculations we used three periods of the lattice in the $\hat{x}$ direction $\left(\Gamma_{x}\right.$ $=$ direction in reciprocal space) and an infinite number of periods in the $\hat{y}$ direction. The physical cavity length, $L_{C}$, was chosen to be approximately two wavelengths of the guided mode and is optimized (to compensate for penetration into the mirror) to provide the highest $Q$ for the guided mode in the case in which the holes extend well into the bottom cladding (approaching the infinite depth regime). The cavity length was reoptimized for each of the three different waveguide geometries analyzed. Of course, as the hole depth changes, so does the mid-gap frequency of the photonic crystal. Thus the longitudinal resonant mode will see varying regions of the photonic band-gap as the hole depth is varied.

We used only three periods in the $\hat{x}$-direction simply because we were interested in small microcavities, not extended modes that penetrate well into the mirror. The photonic crystal was also chosen to be infinite in the $\hat{y}$ direction to eliminate any complications arising from the complex lateral reflectivity of a $2 \mathrm{D}$ photonic crystal, as we wanted to focus on the depth effects. We have limited our study to modes with the electric field polarized inplane (TE). This is because we are interested in

\section{Resonant Cavity Structure}
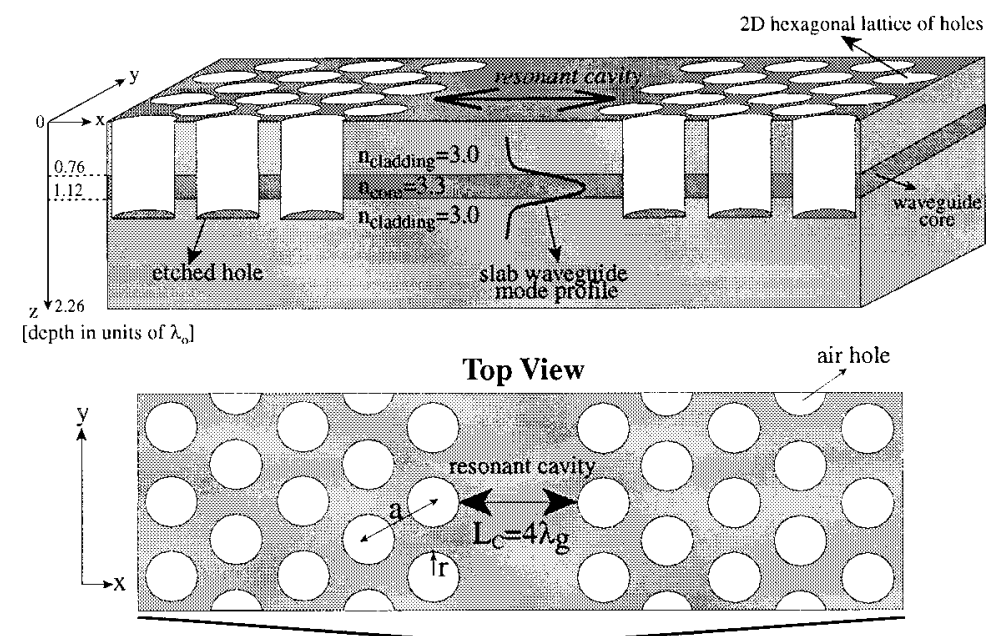

one section of an infinitely periodic structure in $y$-direction

[ $\lambda_{0}=$ wavelength in air, $\lambda_{\mathrm{g}}=$ wavelength in core, $\mathrm{r} / \mathrm{a}=0.4, \mathrm{a} / \lambda_{\mathrm{O}}=0.28$ ]

Fig. 2. The optical cavity is formed from a three-layer slab waveguide and $2 \mathrm{D}$ photonic crystal end mirrors. $\lambda_{0}$ is the wavelength of light in air, $\lambda_{g}$ is the wavelength of light in the core, and the $2 \mathrm{D}$ hexagonal lattice has $r / a=0.4$ and $a / \lambda_{0}=0.28$. 
quantum-well active regions in which the electronic states preferentially couple to the TE modes. This situation closely resembles that of the in-plane semiconductor laser we previously fabricated incorporating 2D photoniccrystal end mirrors. ${ }^{3}$ In Ref. 3 we measured modal reflectivities for the laser end mirrors, which we believe were strongly affected by the finite depth (and variation in depth) of the photonic crystal.

The numerical analysis is performed by means of a uniform resolution $3 \mathrm{D}$ mesh of points used to define the structure. An initial guess for the electromagnetic field is used as a starting point for the calculations. There are no sources of electromagnetic energy, other than the initial field, as the calculations are done for a cold cavity. The initial electromagnetic field, with a broad spectral range, is evolved in time by use of the integral form of Maxwell's equations and finite-difference-time-domain techniques. ${ }^{18,19}$ The resulting time-domain signal is then transformed into the frequency domain by use of a Fourier transform. From this procedure we get a rough estimate of the cavity's frequency response as high- $Q$ modes will be associated with large peaks in the frequency spectrum. We are then able to choose an appropriate spectral filter that overlaps the frequency of the mode we wish to select out. ${ }^{20,21}$ As the mode pattern stabilizes, we fine tune the filter; this reduces its spectral width and repositions the maximum. Once we have selected out a given mode, we measure the $Q$ by calculating the power lost to absorbing boundaries. The absorbing boundaries, formed from an antireflection coating and a good conductor (absorber), ${ }^{22}$ are placed far enough away from the cavity so as not to interfere with the cavity mode.

From the $Q$ and the field pattern we can estimate the modal reflectivity. The field pattern provides us with an effective cavity length, and we can then use the following relation $^{23}$ to find the modal reflectivity:

$$
Q=\omega t_{\mathrm{ph}}=\frac{-2 \pi L_{\lambda_{g}}}{\ln R_{\text {modal }}}
$$

Where $t_{\mathrm{ph}}$ is the photon lifetime in the cavity, $\lambda_{g}$ is the wavelength in the core region of the waveguide, and $L_{\lambda_{g}}$ is the effective cavity length in units of wavelength. Using the field pattern, we estimate the effective cavity length as the distance into the mirror where the magnetic field amplitude decays to $1 / e$, added to the physical cavity length. The effective cavity length is converted to units of wavelength by our counting the number of nodes in the field pattern.

\section{DISCUSSION OF RESULTS}

In Fig. 3 we have plotted cavity $Q$ versus etch depth for the different waveguides studied. For each point in the plot we have selected out the highest $Q$ mode. We used Eq. (1) to determine modal reflectivities for the photonic crystal as the hole depth is varied. For very low $Q$ modes the effective cavity length is not very well defined (as the guided mode is not well contained in the cavity), and the modal reflectivities are essentially zero. A plot showing the dependence of modal reflectivity on hole depth is also shown in Fig. 4.

\section{A. Case (a): Waveguide with Semiconductor Cladding on Both Sides}

From the plot of modal reflectivity in Fig. 4 we see that, indeed, the reflectivity is a strong function of hole depth. The modal reflectivity makes a sharp transition from essentially $0 \%$ to $100 \%$ when the holes are etched through the core and into the bottom cladding. The slope is very steep in this region, which extends over a range of hole depths of $\lambda_{0} / 4$. For very shallow etch depths (just into the core) the modal reflectivity is low because of the small overlap of the photonic crystal and the guided mode. In this regime the modal reflectivity can be increased by adding more and more periods of the photonic crystal; however, the mode volume will still be quite large. This regime can be likened to that of a distributed-feedback laser grating, in which the the optical cavity has a high $Q$ but is distributed over a large longitudinal extent. As the hole depth increases further and the waveguide core becomes more and more perforated, we see only a slight rise in modal reflectivity. Even though the overlap of the

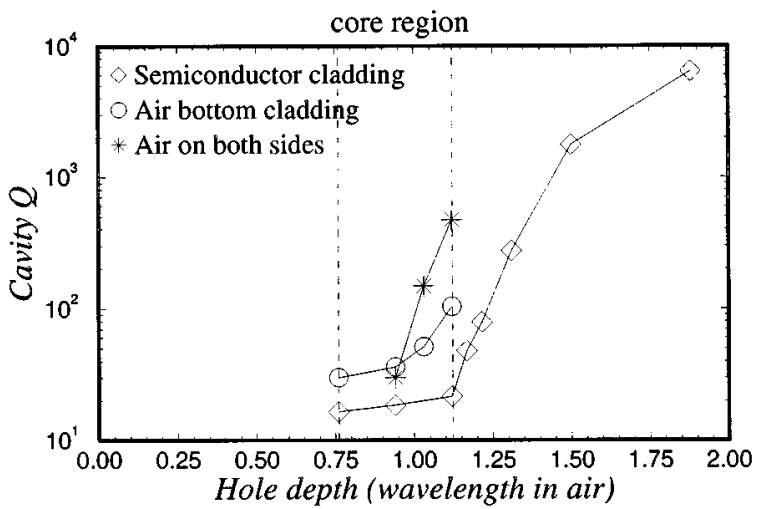

Fig. 3. Cavity $Q$ for various hole depths with a symmetric $(\diamond)$ waveguide with semiconductor on either side of the core, an asymmetric $(O)$ waveguide with air as the bottom cladding, and a symmetric $(*)$ waveguide with air surrounding a semiconductor core.

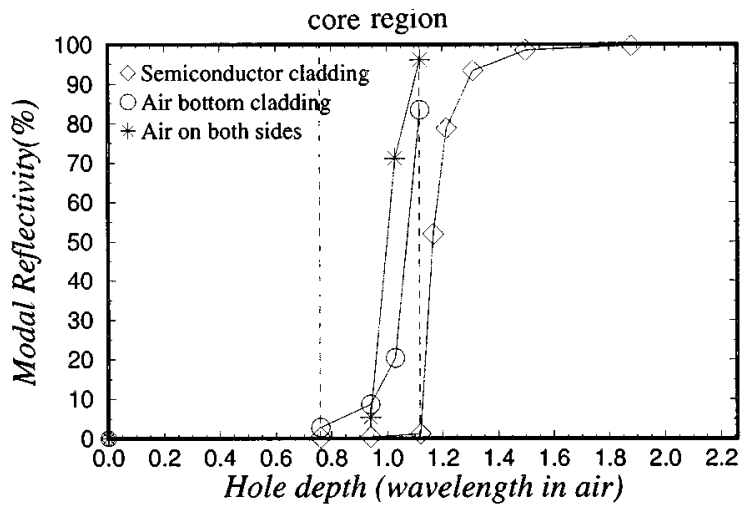

Fig. 4. Modal reflectivity for various hole depths with a symmetric $(\diamond)$ waveguide with semiconductor on either side of the core, an asymmetric $(\bigcirc)$ waveguide with air as the bottom cladding, and a symmetric (*) waveguide with air surrounding a semiconductor core. 


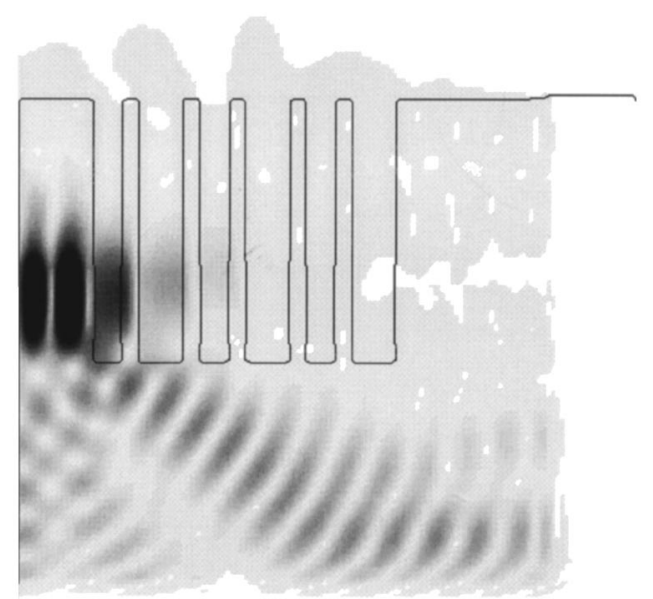

Fig. 5. Semiconductor slab waveguide with photonic crystal holes etched just below the core into the bottom cladding. The magnitude of the magnetic field, enhanced to show cavity losses, is plotted for one side of the symmetrical cavity.

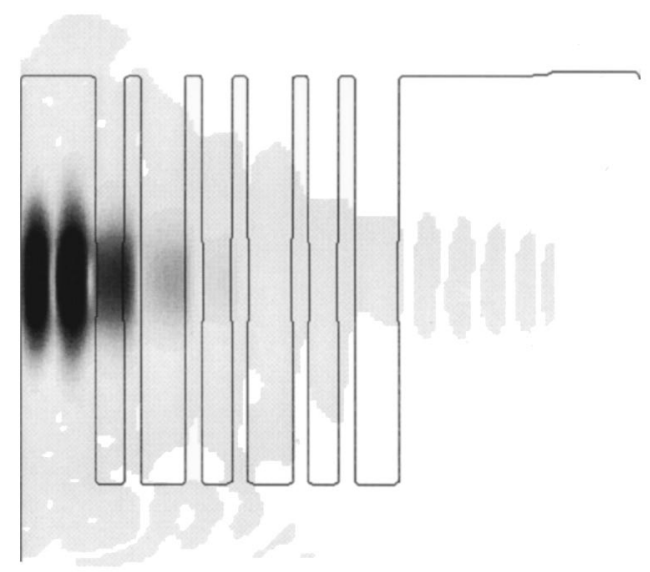

Fig. 6. Semiconductor slab waveguide with photonic crystal holes etched deep into the bottom cladding. The magnitude of the magnetic field, enhanced to show cavity losses, is plotted for one side of the symmetrical cavity.

guided mode and the photonic crystal is substantial in this case, the $2 \mathrm{D}$ photonic crystal is unable to provide the vertical confinement in the third direction necessary for a high- $Q$ mode. When the core is perforated, the effective core refractive index is lowered and the optical mode is no longer guided by total internal reflection at the corebottom cladding interface. This can be most conveniently observed in the plot of the magnitude of the magnetic field shown in Fig. 5, in which the guided mode leaks out of the core and into the substrate as it enters the mirror. The optical losses associated with scattering and leaky waveguiding cannot be reduced by addition of more periods, and thus the modal reflectivity is severely limited for hole depths that do not penetrate the bottom cladding. Finally, when the holes extend into the bottom cladding, the guided mode is confined in the vertical direction as it propagates into, and is reflected by, the photonic crystal. In this regime the modal reflectivity rises close to $100 \%$, and the curve flattens out. A plot of the magnitude of the magnetic field for deep holes is given in Fig. 6, which shows the clean reflection from the mirror and weak transmission into the waveguide at the mirror's output.

\section{B. Case (b): Waveguide with the Bottom Semiconductor Cladding Replaced by Air}

To prevent the light from escaping underneath the core of the waveguide, it is possible to lower the refractive index of the bottom cladding layer in the waveguide structure. Technically this can be done by either a selective chemical etch or a conversion of the bottom cladding layer, or by epitaxially lifting-off the waveguide and placing it on top of a low refractive-index substrate, such as $\mathrm{SiO}_{2}$ or $\mathrm{Al}_{2} \mathrm{O}_{3}$. To modify our model we replaced the bottom cladding with a refractive index of air. The resulting $Q$ 's and modal reflectivities are displayed in Figs. 3 and 4. To our surprise, we discovered that scattering losses from the bottom of the holes results in only a marginal improvement in the modal reflectivities over the structure in case (a). Even if the holes of the photonic crystal extend to the bottom of the core only a modest modal reflectivity of $83 \%$ is attainable. In Fig. 7 the light in the core scatters upward into the remaining cladding layer and eventually escapes the photonic-crystal cavity.

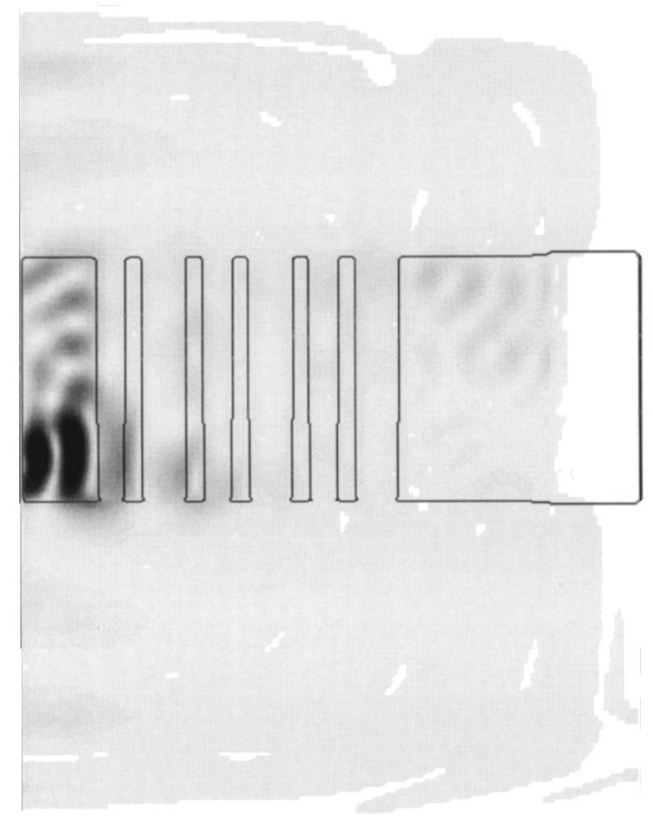

Fig. 7. Semiconductor waveguide with bottom cladding replaced by air. The photonic crystal holes are etched through the core. The magnitude of the magnetic field, enhanced to show cavity losses, is plotted for one side of the symmetrical cavity.

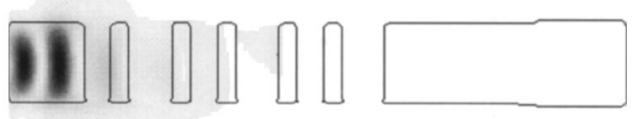

Fig. 8. Semiconductor waveguide core with air on both sides and the photonic crystal holes etched right through the core. The magnitude of the magnetic field, enhanced to show cavity losses, is plotted for one side of the symmetrical cavity. 


\section{Case (c): Waveguide with Air Cladding on Both Sides}

The third and final case that was studied was one in which the waveguide structure consists of a semiconductor core with air on both sides. Such a structure, made popular by whispering-gallery mode lasers, provides very strong vertical mode confinement and reduces many of the losses associated with leaky waveguiding and scattering from the bottom of the etched holes. Again, this structure can be manufactured if the bottom cladding layer is either converted to, or replaced by, a low-index material. The three hole depths analyzed in this case were holes penetrating $1 / 2,3 / 4$, and all the way through the waveguide. In case (c) we saw an improvement in the modal reflectivity to $95 \%$ when the waveguide was completely perforated. The resulting optical mode pattern is shown in Fig. 8, in which little penetration into the photonic crystal is observed.

\section{CONCLUSIONS}

$2 \mathrm{D}$ photonic crystals can be fabricated to form high- $Q$ microcavities. However, light scattering in the vertical direction owing to the finite depth of photonic crystal has to be minimized to yield high-quality $2 \mathrm{D}$ photonic band-gap mirrors. This can be accomplished either with a conventional semiconductor waveguide by definition of deep holes through the core and into the bottom cladding or by confinement of the light in a thin core surrounded by very low-index materials (air). Technologies, such as steam oxidation of AlA's, epitaxial lift-off, and high-resolution lithography and etching methods have become available to fabricate such structures. Along with 3D finitedifference-time-domain modeling capabilities, such as those used in this paper, functional devices based on photonic crystals can now be designed and fabricated.

\section{ACKNOWLEDGMENTS}

The authors thank C. C. Cheng and E. Yablonovitch for helpful discussions. This work was supported by the U.S. Army Research Office under contract DAAH04-96-10389 and the National Science Foundation under contract ECS-9632937.

\section{REFERENCES}

1. E. Yablonovitch, Phys. Rev. Lett. 58, 2059 (1987).

2. M. Boroditsky and E. Yablonvitch, Phys. World 10(7), 25-26 (1997).

3. J. O'Brien, O. Painter, C. C. Cheng, R. Lee, A. Scherer, and A. Yariv, Electron. Lett. 32, 2243 (1996).

4. M. Kanskar, P. Paddon, V. Pacradoui, R. Morin, A. Busch, J. F. Young, S. R. Johnson, J. Mackenzie, and T. Tiedje, Appl. Phys. Lett. 70, 1438 (1997).

5. D. L. Bullock, C. C. Shih, and R. S. Margulies, J. Opt. Soc. Am. B 10, 399 (1993).

6. T. F. Krauss and R. M. De La Rue, Appl. Phys. Lett. 68, 1613 (1996).

7. T. F. Krauss, R. M. De La Rue, and S. Brand, Nature (London) 383, 699 (1996).

8. C. C. Cheng, A. Scherer, V. Arbet-Engels, and E. Yablonovitch, J. Vac. Sci. Technol. B 14, 4110 (1996).

9. T. Hamano, H. Hirayama, and Y. Aoyyagi, in Conference on Lasers and Electro-Optics, Vol. 11 of OSA Technical Digest Series (Optical Society of America, Washington, D.C., 1997), pp. 528-529.

10. G. Feiertag, W. Ehrfeld, H. Freimuth, H. Kolle, H. Lehr, M. Schmidt, M. M. Sigalas, C. M. Soukoulis, G. Kiriakidis, T. Pederson, J. Kuhl, and W. Koenig, Appl. Phys. Lett. 71, 1441 (1997).

11. L. A. Coldren and S. W. Corzine, Diode Lasers and Photonic Integrated Circuits (Wiley, New York, 1995).

12. T. Baba and T. Matsuzaki, Jpn. J. Appl. Phys., Part 1 35, 1348 (1996).

13. K. Sakoda, Phys. Rev. B 52, 8992 (1995).

14. E. M. Purcell, Phys. Rev. 69, 681 (1946).

15. G. Björk, H. Heitmann, and Y. Yamamoto, Phys. Rev. A 47, 4451 (1993).

16. G. il Kweon and N. M. Lawandy, Opt. Commun. 118, 388 (1995).

17. J. D. Joannopoulos, R. D. Meade, and J. N. Winn, Photonic Crystals (Princeton University, Princeton, N.J., 1995).

18. K. S. Yee, IEEE Trans. Antennas Propag. 14, 302 (1966).

19. M. Bartsch, M. Dehler, M. Dohlus, F. Ebeling, P. Hahne, R. Klatt, F. Krawczyk, M. Marx, Z. Min, T. Pröpper, D. Schmitt, P. Schütt, B. Steffen, B. Wagner, T. Weiland, S. G. Wipf, and H. Wolter, Comput. Phys. Commun. 72, 22 (1992).

20. D. H. Choi and W. J. R. Hoefer, IEEE Trans. Microwave Theory Tech. 34, 1464 (1986).

21. Z. Bi, Y. Shen, K. Wu, and J. Litva, IEEE Trans. Microwave Theory Tech. 40, 1611 (1992).

22. K. Chamberlain and L. Gordon, IEEE Trans. Electromagn. Compat. 37, 210 (1995).

23. A. Yariv, Optical Electronics, 4 th ed. (Saunder College, Orlando, 1991). 